\title{
Improved $R$-factors for diffraction data analysis in macromolecular crystallography
}

Kay Diedrichs and P. Andrew Karplus

Nature Structural Biology 4, no. 4, 269-275 (1997).

The Rsym definition on p. 269 should read:

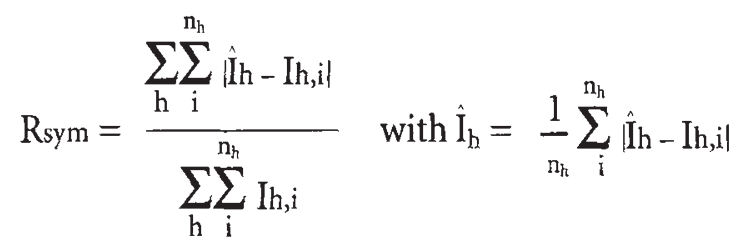

(thanks to Clemens Vonthein for finding the missing summation sign)

The first sentences of the legend for Fig. 2 should read: "The behaviour of data quality indicators as a function of multiplicity. Solid lines are used for the following three indicators: $R_{s y m}$ (filled triangle); PCV (filled square); $R_{\text {mrgd.F }}$ (open square). Dashed lines are used for $\mathrm{R}_{\text {meas }}(\mathrm{X}) ; \mathrm{R}_{\text {mrgd-I }}$ (open circle); $<1 / \sigma_{\mathrm{I}}>$ (closed circle)."

In Fig. 2, it should be $\left\langle 1 / \sigma_{1}>(\right.$ not $\left.<1 / s\rangle\right)$.

On p. 272, there are only space groups of the form $\mathrm{P} 4_{(0,1,2,3)} 2_{(0,1)} 2\left(\right.$ not $\left.P 4_{(0,1,2,3)} 2_{(0,1)} 2_{(0,1)}\right)$.

On p. 275 (box), several typesetting errors make understanding difficult:

line 2: "true intensity $\tilde{\mathrm{I}}_{\mathrm{h}}$ " (note the tilde).

lines 9-12: "As the $I_{h, i}$ and $I_{h, j}\left(j \neq i\right.$ ) (note: not jfii) are independent, the variance $s \Delta_{h}^{2}$ of the $\Delta_{h, i}$ can be calculated as the sum of the variances of the two terms on the right-hand side of the last equation:

$s_{\Delta_{\mathrm{h}}}{ }^{2}=\left(\frac{\mathrm{n}_{\mathrm{h}}-1}{\mathrm{n}_{\mathrm{h}}}\right)^{2} s_{\mathrm{h}}^{2}+\left(\mathrm{n}_{\mathrm{h}}-1\right) \frac{1}{\mathrm{n}_{\mathrm{h}}^{2}} s_{\mathrm{h}}^{2}=\frac{\mathrm{n}_{\mathrm{h}}-1}{\mathrm{n}_{\mathrm{h}}} s_{\mathrm{h}}{ }^{2}$ " (note: this defines $s \Delta_{\mathrm{h}}^{2}$, not $\left.s_{\mathrm{h}}{ }^{2}\right)$

The final paragraph on p. 275 should start as follows:

Relation of $R_{\text {meas }}, P C V$, and $R_{\mathrm{mrgd}-\mathrm{I}}$ to the average $I / \sigma$ ratio of the measured and reduced data

Data reduction programs calculate the estimates of the variance of $\sigma_{h, i}{ }^{2}$ for each $\mathrm{I}_{\mathrm{h}, \mathrm{i}}$ from counting statistics and background level, and most report the $\langle\mathrm{I} / \sigma\rangle$ ratio as a funciton of resolution. From a statistical standpoint, the sample variance $s_{\mathrm{h}}{ }^{2}$ of the $\mathrm{I}_{\mathrm{h}, \mathrm{i}}$ should be consistent with these $\sigma_{\mathrm{h}, \mathrm{i}}{ }^{2}$, if no systematic differences between observations exist ${ }^{20}$. In this case, it follows from (9) that both Rmeas (eqn. 2 ) and PCV (eqn. 3) are related to the inverse of the average signal-to-noise ratio $\left\langle\mathrm{I} / \sigma_{1}>\right.$ of the observations. [Note the $s$ versus $\sigma$ confusion in the article as printed]

Update: Reference 10 is Weiss, M.S. \& Hilgenfeld, R. J. Appl. Cryst. 30, 203-205 (1997).

\section{Solution structure of an rRNA methyltransferase (ErmAM) that confers macrolide-lincosamide-streptogramin antibiotic resistance}

Liping Yu, Andrew M. Petros, Arndt Schnuchel, Ping Zhong, Jean M. Severin, Karl Walter, Thomas F. Holzman and Stephen W. Fesik

Nature Structural Biology 4, no. 6, 483-489 (1997).

Owing to a printing error, Figures $2-5$ do not agree with the text. Helices $\alpha 5-\alpha 11$ in the text correspond to $\alpha 3-\alpha 9$ in the published figures, $\alpha 3$ to residues $62-65$ between $\beta 3$ and $\beta 4$, and $\alpha 4$ to residues $85-88$ between $\beta 4$ and $\beta 5$. The correct figures can be obtained in the reprints of this article from S.W. Fesik (fesik@steves. abbott.com). 